\title{
Total Synthesis as a Resource in the Drug Discovery: The First In Vivo Evaluation of Panaxytriol and its Derivatives
}

\author{
Heedong Yun ${ }^{\dagger}$, Ting-Chao Chou ${ }^{\ddagger}$, Huajin Dong ${ }^{\ddagger}$, Yue-ming $\mathrm{Li}^{+}$, and Samuel J. Danishefsky ${ }^{\dagger,}, *$ \\ ${ }^{\dagger}$ Department of Chemistry, Columbia University, 3000 Broadway, New York and ${ }^{\S}$ Laboratory for Bioorganic Chemistry, ${ }^{\ddagger}$ Preclinical \\ Pharmacology Core Facility, and ${ }^{+}$Laboratory of Biochemistry and Pharmacology, Sloan-Kettering Institute for Cancer Research, \\ 1275 York Ave., New York, N.Y. 10021 (USA) \\ E-mail: s-danishefsky@ski.mskcc.org
}

Supporting Information

Table of contents

1. general procedure - S2

2. ${ }^{1} \mathrm{H}$ and ${ }^{13} \mathrm{C}$ nmr of $5-\mathrm{S} 3, \mathrm{~S} 4$

3. ${ }^{1} \mathrm{H}$ and ${ }^{13} \mathrm{C}$ nmr of $\mathbf{1}-\mathrm{S} 5$, S6

4. ${ }^{1} \mathrm{H}$ and ${ }^{13} \mathrm{C}$ nmr of $\mathbf{1 2}-\mathrm{S} 7, \mathrm{~S} 8$

5. ${ }^{1} \mathrm{H}$ and ${ }^{13} \mathrm{C}$ nmr of $\mathbf{1 3}$ - S9, S10

6. ${ }^{1} \mathrm{H}$ and ${ }^{13} \mathrm{C}$ nmr of $\mathbf{1 4}-\mathrm{S} 11, \mathrm{~S} 12$ 


\section{General Procedures}

All commercial materials were used without further purification unless otherwise noted. THF, diethyl ether and dichloromethane used as reaction solvents were obtained from a dry system (alumina) and used without further drying. All reactions were performed under a positive pressure of argon atmosphere in flame dried vessel. ${ }^{1} \mathrm{H}$ NMR spectra were obtained on 300 and $400 \mathrm{MHz}$ instruments and are reported in parts per million $(\delta)$ using residual non deuterated solvent as internal reference. ${ }^{13} \mathrm{C}$ NMR spectra were recorded on 75 $\mathrm{MHz}$ and $125 \mathrm{MHz}$ instruments and reported in parts per million ( $\delta$ ) using residual non deuterated solvent as internal reference. IR spectra were taken as a thin film on a FT-IR Spectrometer. Optical rotations were recorded on a polarometer using a $1 \mathrm{dm}$ cell at the reported temperatures and concentrations. High resolution mass spectra were recorded on mass spectrometer. Liquid column chromatography was performed using forced flow of a mixture of solvents on silica gel 60 (40-63mm).

Check the following reference for characterizations of synthetic intermediates for panaxytriol:

1. Yun, H.; Danishefsky, S. J. J. Org. Chem. 2003, 68, 4519. 


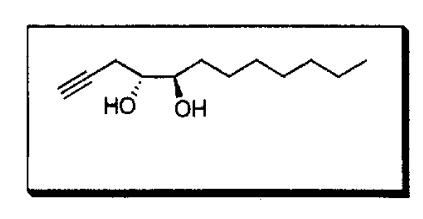

\section{W}

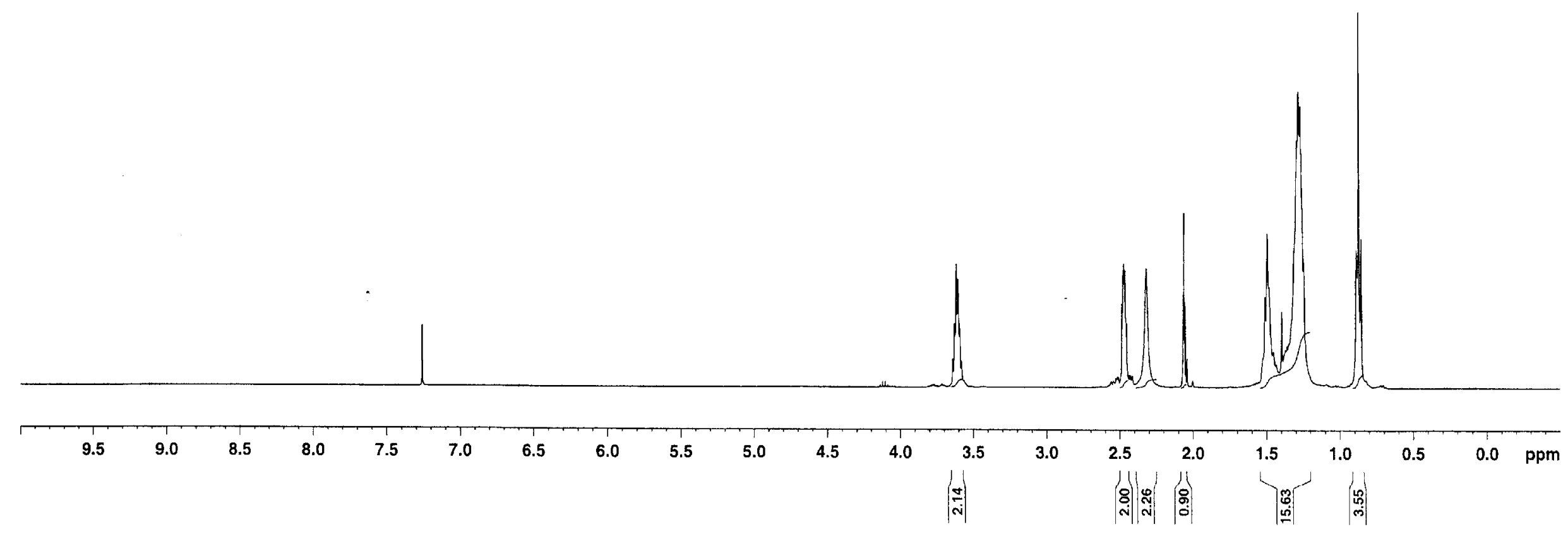



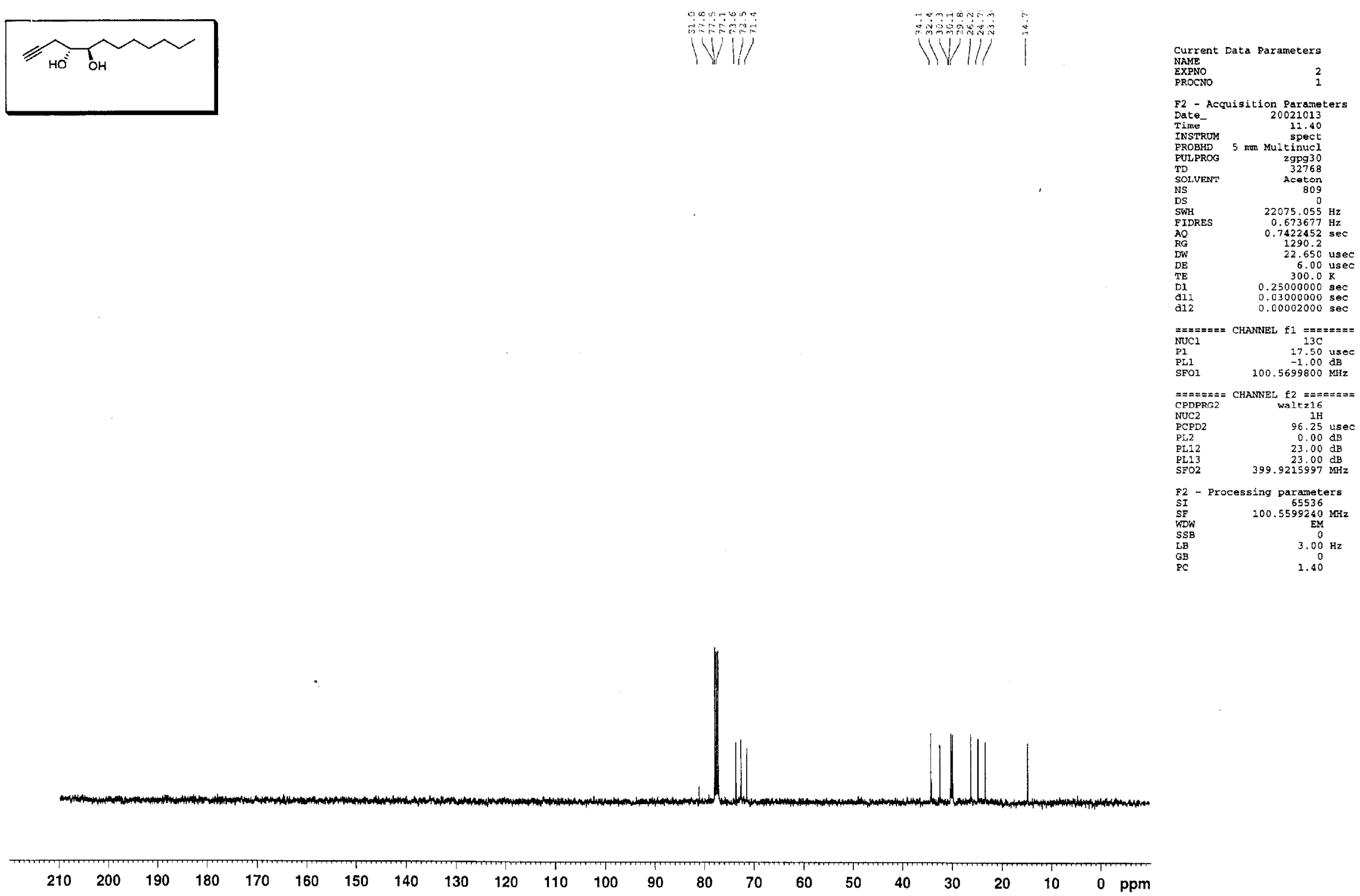

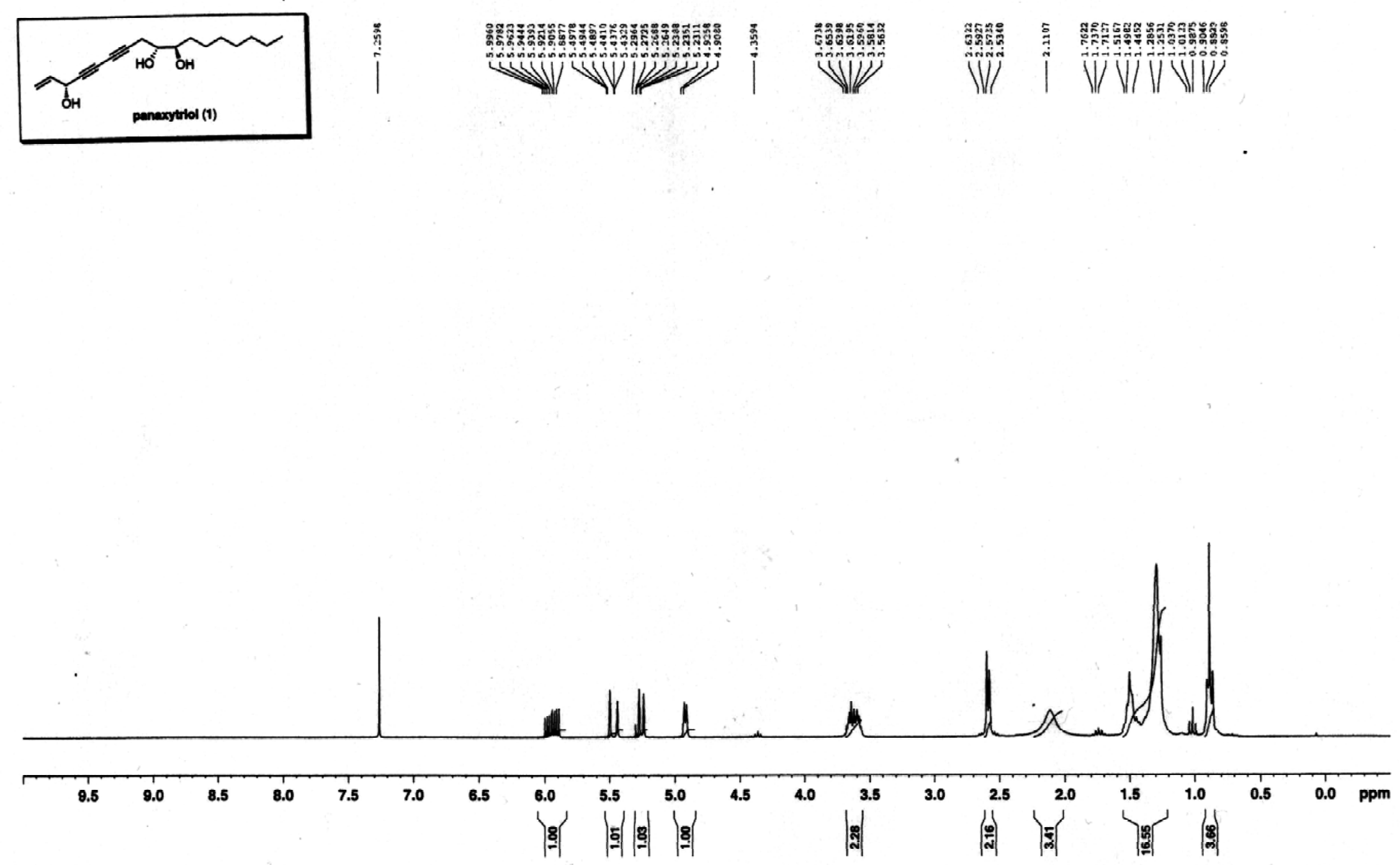
carbon NMR

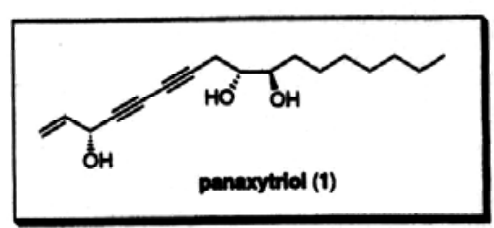

:

क्रिक

W/11/11

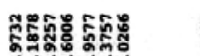

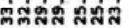

$\|$ |

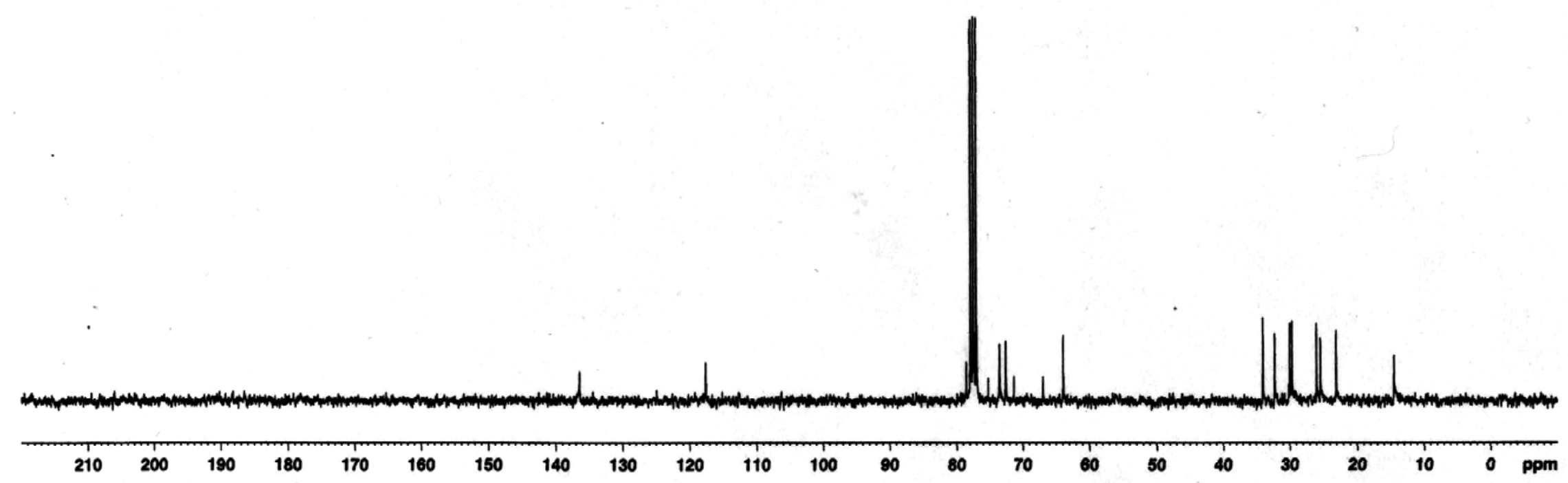


辛 4 L

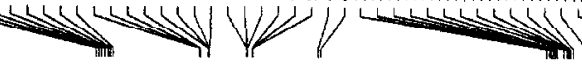
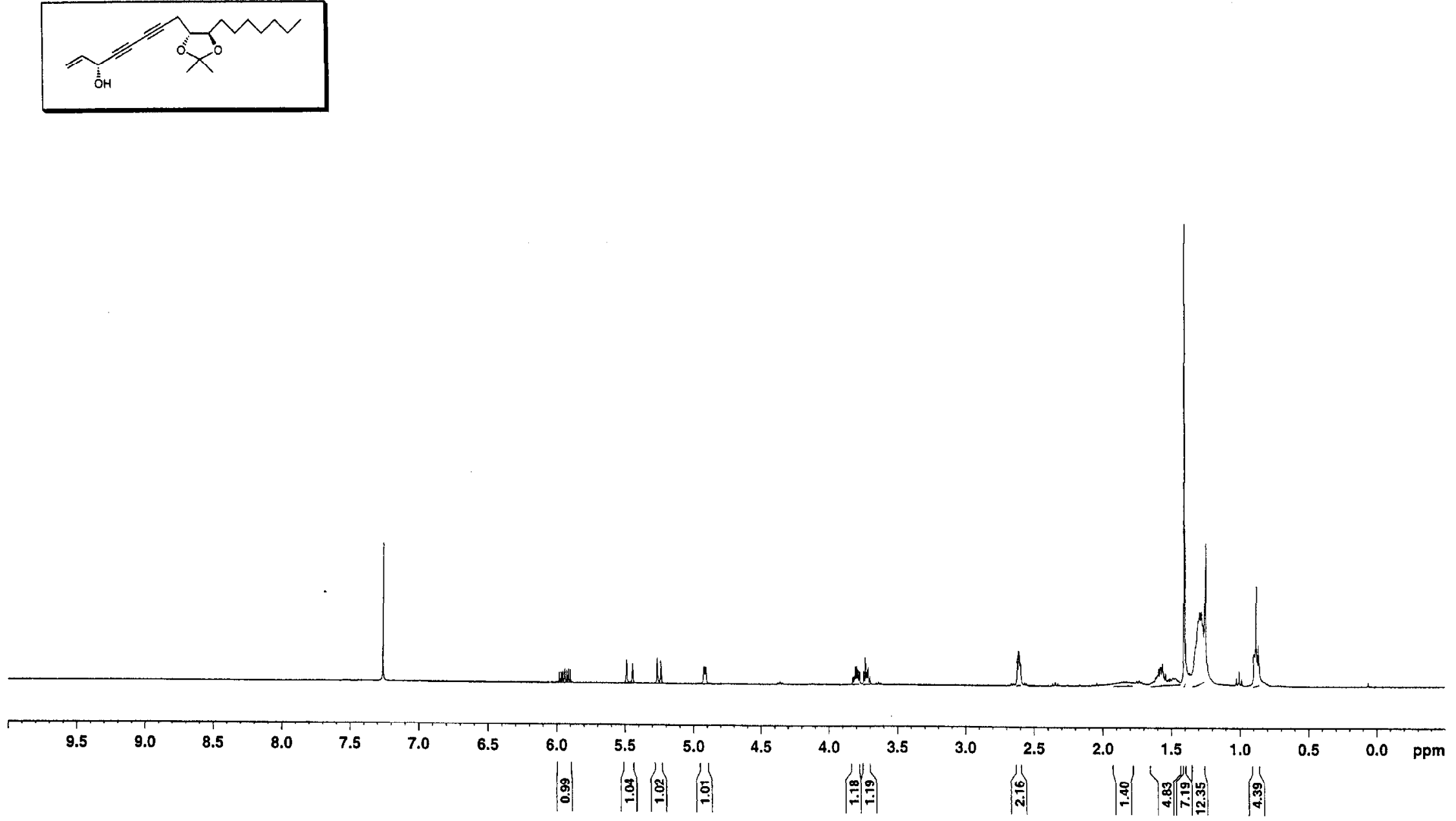


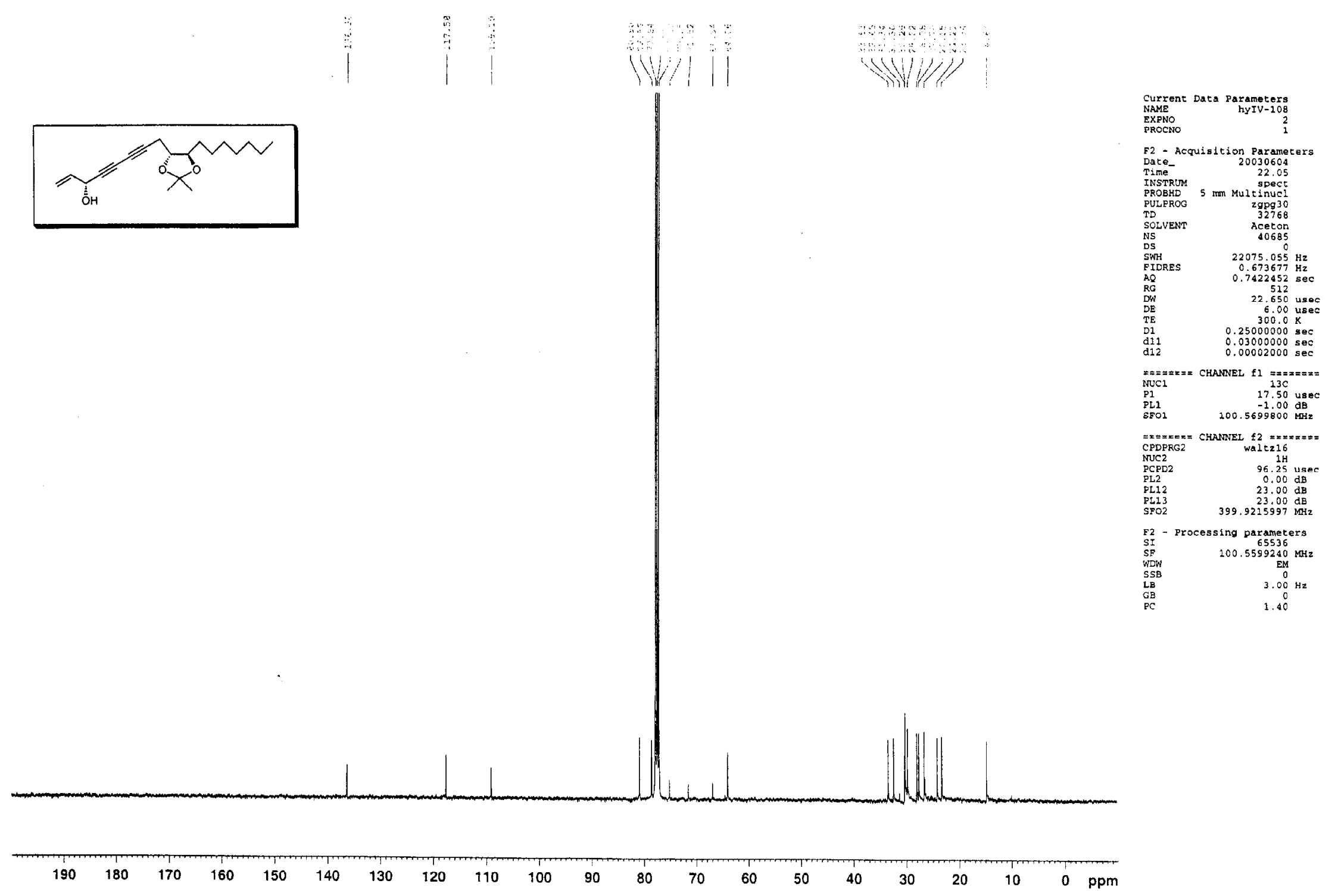



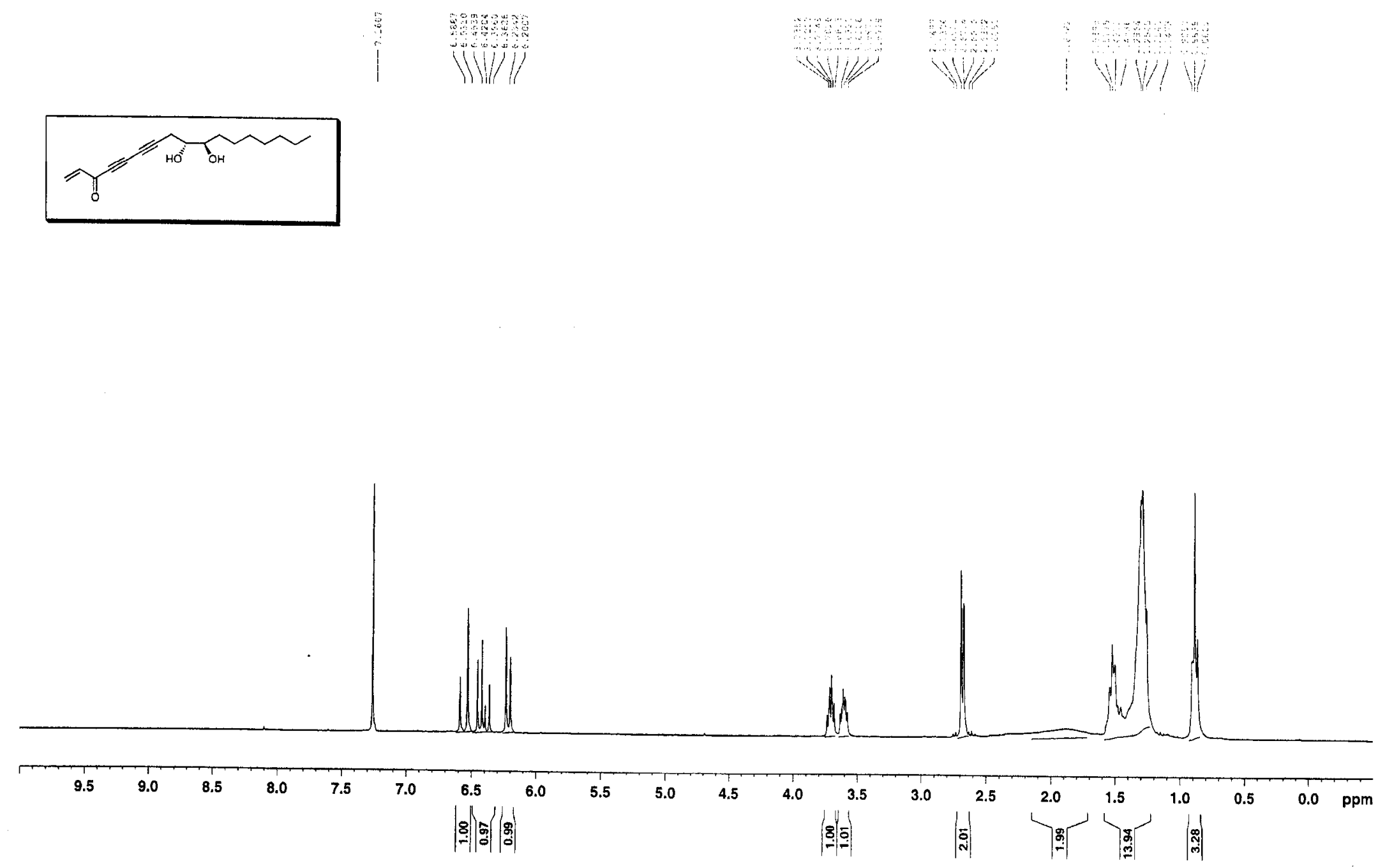

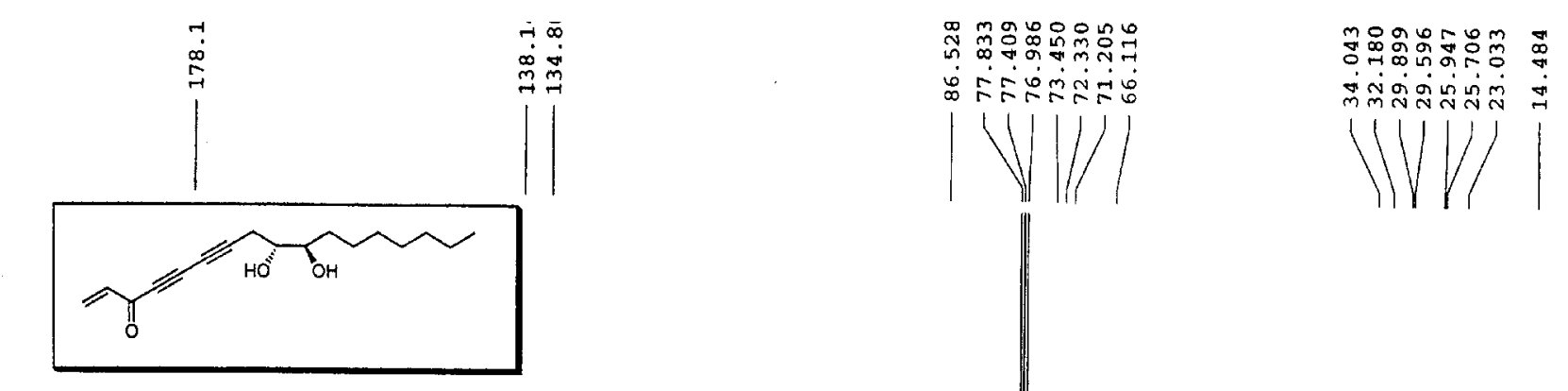

Current Data Parameters

NAME hYIV-130

EXPNO

DROCNO

2
1

F2 - Acquisition Parameters

Time- 20030616

INSTRU

PROBHD 5 min Multinucl

PULPROG

TD

gpg 30

SOLVENT

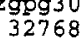

DS

SWH

FIDRES

$A Q$
$R G$

$\mathrm{DW}$

$\mathrm{DE}$

DI

8172

$23809.523 \mathrm{~Hz}$

$0.6881780 \mathrm{sec}$

4597.6

21.000 usec 21.00 usec

$300.0 \mathrm{~K}$

$0.03000000 \mathrm{sec}$ $0.00002000 \mathrm{sec}$

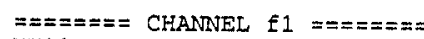
NUC1

P1 1

$13 C$
11.40
5.00 usec

PL1

$==x=x= \pm=$ CHANNEL f $2=x=====$

CPDPRG2 waltz 16

100.00 usec

PCPD

100.00 used
$3.00 \mathrm{~dB}$

PL12
PL13

$29.70 \mathrm{~dB}$
$29.70 \mathrm{~dB}$

SFO2 $300.1312005 \mathrm{MHz}$

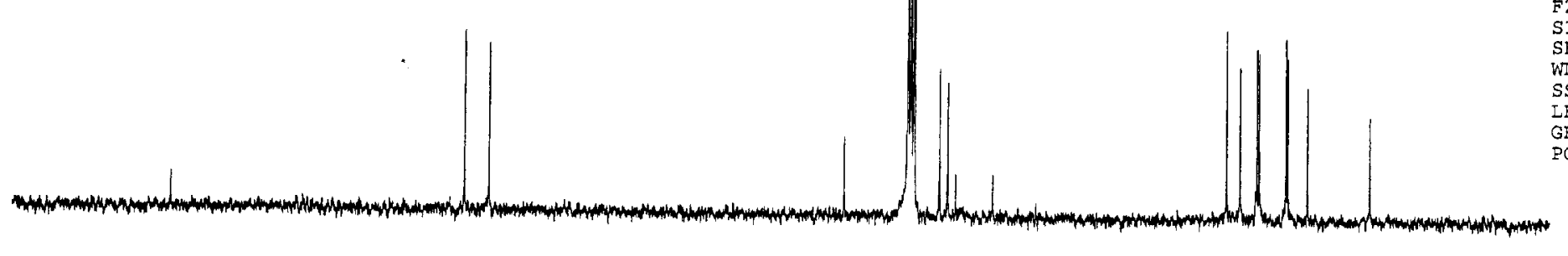

F2 - Processing parameters

SI $\quad 65536$

WDW

SSB

$\mathrm{GB}$

EM
0
$3.00 \mathrm{~Hz}$
0
1.40

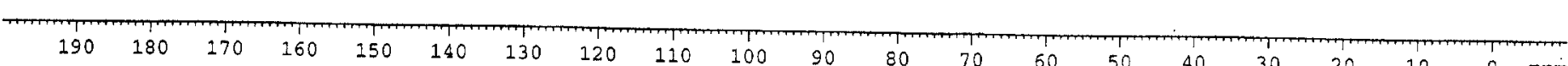


proton
hyIV-254<smiles>C=CC(=O)C#CC#CC[C@H](OC(C)(C)C)C(O)CCCCCCC</smiles>

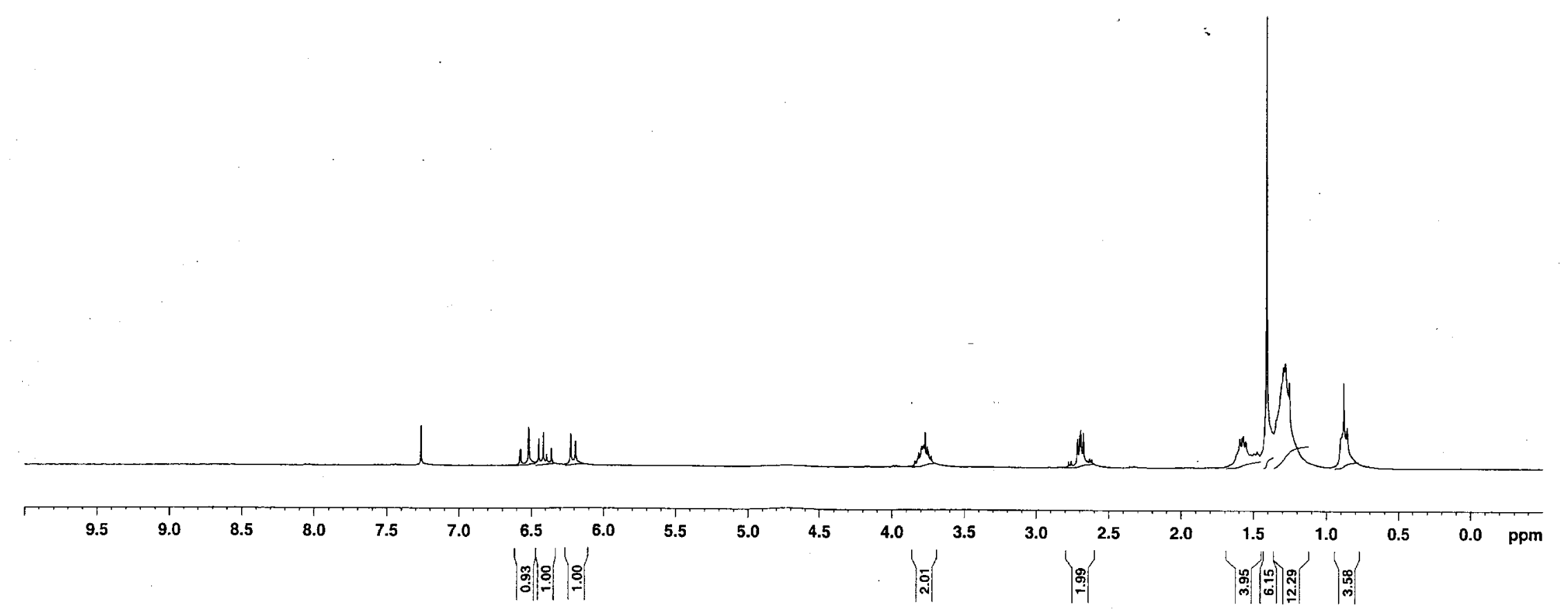




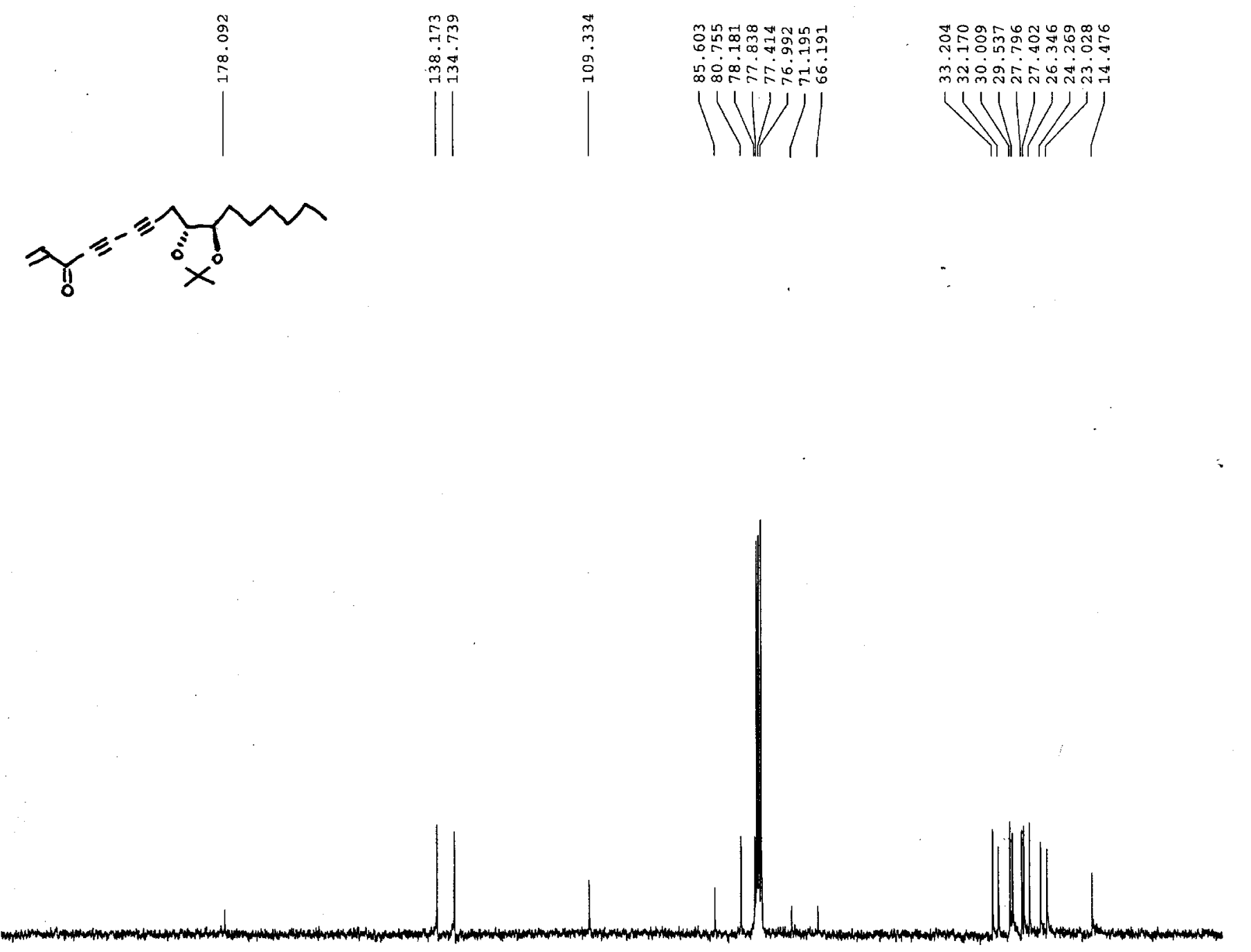

$\begin{array}{lr}\text { Current Data } & \text { Parameters } \\ \text { NAME } & \text { hYIV-254 } \\ \text { EXPNO } & 2 \\ \text { PROCNO } & 1\end{array}$

F2 - Acquisition Parameters

$\begin{array}{lr}\text { Date__ } & 20030815 \\ \text { Time } & 12.43\end{array}$

$\begin{array}{ll}\text { Time } & 12.43 \\ \text { INSTRUM } & \text { spect }\end{array}$

INSTRUM
PROBHD $5 \mathrm{~mm}$ Multinucl

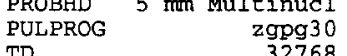

$\begin{array}{lr}\text { TD } & 32768 \\ \text { SOLVENT } & \text { CDC13 } \\ \text { NS } & 2669\end{array}$

2669
0

SWH $\quad 23809.523 \mathrm{~Hz}$

FIDRES $\quad 0.726609 \mathrm{~Hz}$

$\begin{array}{lr}\text { AQ } & 0.6881780 \mathrm{sec} \\ \text { RG } & 3251\end{array}$

$\begin{array}{lr}\text { RG } & 3251 \\ \text { DW } & 21.000 \text { usec }\end{array}$

DE $\quad 21.00$ usec

$0.69999999 \mathrm{sec}$

d11 $\quad 0.03000000 \mathrm{sec}$

d12 $\quad 0.00002000 \mathrm{sec}$

$=======$ CHANNEL $11=======$
NUC1
$13 \mathrm{C}$

$\begin{array}{lr}\text { NUC1 } 113 \mathrm{C} \\ \text { P1 } & 11.40 \text { usec }\end{array}$

55.01
SFO1

$=======$ CHANNEL f $2=======$ CPDPRG2 CHANEL waltz16

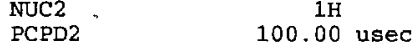

$\begin{array}{lr}\text { PL2 } & 100.00 \mathrm{us} \\ \mathrm{BL} & 3.00 \mathrm{~dB}\end{array}$

$\begin{array}{ll}\text { PL12 } & 29.70 \mathrm{~dB} \\ \text { PL13 } & 29.70 \mathrm{~dB}\end{array}$

SF02 $300.1312005 \mathrm{MHz}$

F2 - processing parameters

SI $\quad 65536$

\begin{tabular}{l|l} 
SF & 75.46771
\end{tabular}

SSB

SSB
LB
GB

PC

$3.00 \mathrm{~Hz}$

1.00

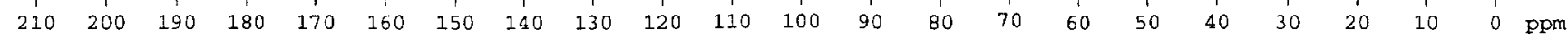

